\title{
Definition and Extraction of Lip Protrusion Based on the Facial Skeleton Data
}

\author{
Xiaosheng Pan ${ }^{1}$, Menghan Zhang ${ }^{2}$, Wee Chung Liew ${ }^{3}$ \\ ${ }^{1}$ Shanghai Normal University, Shanghai 200234; \\ ${ }^{2}$ Fudan University, Shanghai 200433; \\ ${ }^{3}$ Griffith University, Queensland, Australia; \\ itol_xs@shnu.edu.cn, hansonmenghan@163.com, a.liew@griffith.edu.au
}

\begin{abstract}
The current paper discussed the definition of lip protrusion and proposed an effective method to measure the lip protrusion. Because the movement patterns for upper lip and low lip were distinct, the authors defined the low and upper lip protrusion respectively as the Euclidean distance between the lip edge and incisors. Three-dimensional lip data were obtained by catching the movement trajectory of the reference points pasted on the forehead and mandible through motion capture. The singular value decomposition(SVD) method was applied to rectify the positional information of lower and upper incisors, and this rectified information and the coordinates of the lip edge data were used to calculate the lip protrusion. The method proposed in this paper was tested in 3D lip data, and it was also proved to be effective in analyzing two-dimensional lip data.
\end{abstract}

Index Terms: lip protrusion; Singular Value Decomposition; rigid motion; Euclidean distance 